\title{
Multiple Adaptable Mechanisms Early in the Primate Visual Pathway
}

\author{
Neel T. Dhruv, Chris Tailby, Sach H. Sokol, and Peter Lennie \\ Center for Neural Science, New York University, New York, New York 10003
}

We describe experiments that isolate and characterize multiple adaptable mechanisms that influence responses of orientation-selective neurons in primary visual cortex (V1) of anesthetized macaque (Macaca fascicularis). The results suggest that three adaptable stages of machinery shape neural responses in V1: a broadly tuned early stage and a spatio-temporally tuned later stage, both of which provide excitatory input, and a normalization pool that is also broadly tuned.

The early stage and the normalization pool are revealed by adapting gratings that themselves fail to evoke a response from the neuron: either low temporal frequency gratings at the null orientation or gratings of any orientation drifting at high temporal frequencies. When effective, adapting stimuli that altered the sensitivity of these two mechanisms caused reductions of contrast gain and often brought about a paradoxical increase in response gain due to a relatively greater desensitization of the normalization pool.

The tuned mechanism is desensitized only by stimuli well matched to a neuron's receptive field. We could thus infer desensitization of the tuned mechanism by comparing effects obtained with adapting gratings of preferred and null orientation modulated at low temporal frequencies.

\section{Introduction}

Prolonged viewing of a high contrast pattern reduces a viewer's sensitivity to that and similar patterns (Blakemore and Campbell, 1969; Movshon and Blakemore, 1973; Graham, 1989). For example, prolonged viewing of a grating results in a loss of sensitivity to gratings of similar orientation and spatial frequency, but not to gratings of substantially different orientation or spatial frequency (Blakemore and Campbell, 1969; Movshon and Blakemore, 1973). The presumed physiological locus of this is primary visual cortex (V1), where neurons first exhibit orientation tuning (Hubel and Wiesel, 1959, 1962).

For the behavior of V1 neurons to account for the perceptual observations, the response of a cell to a test stimulus should be reduced following prolonged exposure to high-contrast patterns that drive the cell well, but should be unaffected by exposure to stimuli that do not drive it. Physiological studies (Maffei et al., 1973; Vautin and Berkley, 1977; Movshon and Lennie, 1979; Albrecht et al., 1984; Ohzawa et al., 1985; Maffei et al., 1986; Carandini et al., 1998) have generally found that prolonged stimulation

\footnotetext{
Received Feb. 17, 2011; revised Aug. 11, 2011; accepted Aug. 29, 2011.

Author contributions: N.T.D., C.T., S.H.S., and P.L. designed research; N.T.D., C.T., S.H.S., and P.L. performed research; N.T.D. analyzed data; N.T.D., C.T., S.H.S., and P.L. wrote the paper.

This work was supported by NIH Grants EY 04440 and EY 13079. We thank N. Majaj for assistance during recording and helpful discussion.

Correspondence should be addressed to Neel T. Dhruv at his present address: Institute of Ophthalmology, University College London, 11-43 Bath Street, London EC1V 9EL, UK. E-mail: n.dhruv@ucl.ac.uk.

C. Tailby's present address: National Vision Research Institute of Australia, Carlton, Victoria, 3053, Department of Optometry and Vision Sciences and Psychological Sciences, The University of Melbourne, Parkville, Victoria, 3052, Australia, and Melbourne Brain Centre, Florey Neuroscience Institutes, Heidelberg, Victoria, Australia.

P. Lennie's present address: Center for Visual Science and the Department of Brain and Cognitive Sciences, University of Rochester, Rochester, NY 14627.

DOI:10.1523/JNEUROSCI.0890-11.2011

Copyright $\odot 2011$ the authors $\quad 0270-6474 / 11 / 3115016-10 \$ 15.00 / 0$
}

with high contrast patterns reduces the contrast sensitivity of neurons in a pattern-selective manner (for review, see Kohn, 2007), but two studies of adaptation in V1 neurons have suggested attributes that are neither easily explained by, nor easily reconciled with, their presumed role in perceptual adaptation. First, prolonged exposure to a high contrast pattern that drives a cell well can lead to strengthened rather than weakened responses (Sclar et al., 1989). Second, V1 neurons in cat can be adapted by prolonged exposure to stimuli to which they do not respond (Crowder et al., 2006).

The effects of contrast adaptation on neural mechanisms are poorly understood, mostly because in different cells adaptation can cause quite different changes in the shapes of the contrast response function, and in any given cell different adapters can give rise to surprisingly diverse effects on response. In this paper we demonstrate and characterize the phenomenological variability of adaptation effects in macaque $\mathrm{V} 1$, and show how it can be derived from a simple model of adaptation in the early visual pathway.

\section{Materials and Methods}

We made single-unit recordings from primary visual cortex in 10 anesthetized, paralyzed male monkeys (Macaca fascicularis). The experiments described here were part of a larger set of experiments performed on these animals. All experiments conformed to the New York University Animal Care and Use Committee and NIH guidelines.

Preparation. Animals were initially sedated with ketamine $(10 \mathrm{mg} / \mathrm{kg}$, i.m.). In addition, atropine sulfate $(0.1 \mathrm{mg} / \mathrm{kg}$, i.m. $)$ was administered to reduce mucous secretions. Supplementary doses of ketamine were administered when deemed necessary because of animal reactivity during initial surgery. The legs and arms were shaved to allow for placement of electrocardiogram (ECG) electrodes and a noninvasive blood pressure cuff. The saphenous veins were cannulized, through which saline, anesthetic and paralytic were administered for the duration of the experi- 
ment. Anesthesia was maintained through the balance of the preparation time with thiopental sodium $(10 \mathrm{mg} / \mathrm{kg}$ initially, followed by quarter doses every $20 \mathrm{~min}$ ). Animals were intubated and then placed in a stereotaxic frame. A craniotomy was performed and a small durotomy made over the area just posterior to the lunate sulcus to allow for recording from the primary visual cortex in the lower visual field between $2^{\circ}$ and $7^{\circ}$ eccentric to the foveae. The animals were switched to an infusion mixture of Normosol-R and dextrose $(2.5 \%)$. Anesthesia was maintained with sufentanil citrate $\left(4-15 \mu \mathrm{g} \cdot \mathrm{kg}^{-1} \cdot \mathrm{h}^{-1}\right)$ and paralysis induced with vecuronium bromide $\left(100 \mu \mathrm{g} \cdot \mathrm{kg}^{-1} \cdot \mathrm{h}^{-1}\right)$. Electroencephalogram (EEG) electrodes were attached to the skull to monitor general brain activity. Vital signs such as ECG, EEG and blood pressure were monitored continuously through the duration of the experiment. In addition, ventilation volume and rate were adjusted to maintain the end tidal $\mathrm{CO}_{2}$ near 35 $\mathrm{mmHg}$. Internal body temperature was maintained near $37^{\circ} \mathrm{C}$ by means of an electric heating blanket and rectal temperature probe. The pupils were dilated with topical application of atropine sulfate (1\%) and the eyes were protected with a topical antibiotic (Neocidin) and then with noncorrective gas-permeable contact lenses. Foveal locations were mapped and refraction corrected with a reversing ophthalmoscope and supplementary external lenses. Subsequently, the refraction was verified and adjusted, if needed, by maximizing the spatial acuity of single cells. A broad-spectrum antibiotic (bicillin 300,000 U) and a steroid to control inflammation (dexamethasone $2 \mathrm{mg}$ ) were administered (intramuscularly) each day.

Physiological recording, stimulus presentation and measurement of contrast response. Electrodes (Merrill-Ainsworth or Thomas) were advanced through the durotomy and into V1. Analog voltage signals were amplified, filtered and sampled at $44.1 \mathrm{kHz}$. Putative spikes were displayed on a window and a template set to select spikes, which were then recorded with an accuracy of $0.1 \mathrm{~ms}$.

Stimuli were presented on a calibrated CRT monitor (Eizo T966, refresh rate 90 or $120 \mathrm{~Hz}$, mean luminance $\sim 50 \mathrm{~cd} / \mathrm{m}^{2}$; Iiyama Vision Master Pro, refresh rate $200 \mathrm{~Hz}$, mean luminance $\sim 35 \mathrm{~cd} / \mathrm{m}^{2}$ ). After a cell was isolated, we performed initial tuning experiments using sinusoidal gratings to determine, in order, the cell's orientation, direction, spatial-frequency, temporal-frequency, chromatic, and size selectivity based on firing-rate responses. The last of these measures enabled us to characterize the strength of any suppressive surround. If a cell was strongly orientation tuned (possessing a null orientation), we measured the contrast response of that cell during different adaptive states.

We measured the responses of cells to drifting achromatic sinusoidal gratings of optimal spatial configuration, at six different contrasts, ranging logarithmically between 0.03 and 1.0, plus a blank screen. The mean luminance was constant. Stimuli were pseudo-randomly presented for at least 10 repeats. We collected contrast response functions before, during, and after at least $5 \mathrm{~min}$ recovery from adaptation. We refer to the before and after measurements as "control" runs; we refer to those made during adaptation as "adaptation" runs. If the post-recovery control run did not match the pre-adaptation control run, both the adaptation and the postrecovery control runs were excluded from analysis. For each neuron, we averaged the contrast responses from all control runs and compared this "unadapted" response with those obtained during the different adaptation runs. We induced the adaptation by exposing the cell to the full contrast adapter for $30 \mathrm{~s}$, after which we measured the cell's response to half-second probes interleaved with five seconds of "top-up" adaptation with half-second gaps between the adapter and the probe. The adapter was a full contrast grating of optimal size and spatial-frequency in adaptation runs and a blank screen in control runs. This procedure ensured that responses were collected in a consistent adaptation state. The probe stimuli in control and adaptation runs were always drifting gratings of optimal orientation, spatial frequency, temporal frequency and aperture size.

Model of adaptable mechanisms. To understand the effects of prolonged stimulation on neural response we adopt a model that consists of three signal-processing stages (generally following the methods of Heeger, 1992; Carandini et al., 1997) (Fig. 1). The first is an early-stage mechanism that is broadly tuned in space and time, comprising multiple cell types (see below). The second is an orientation- and spatial-

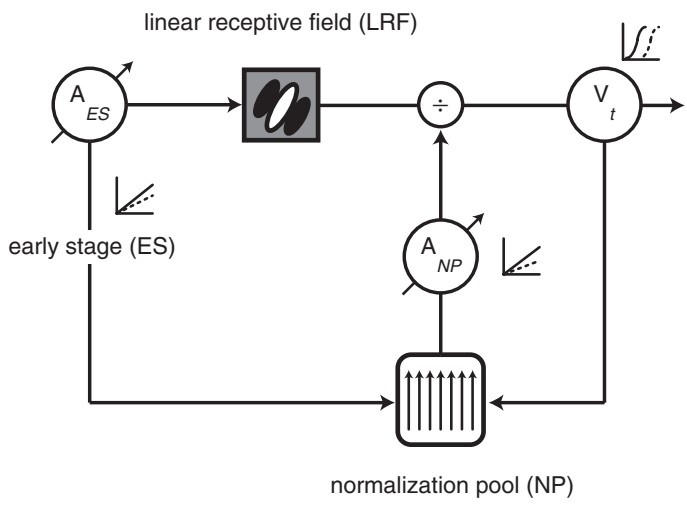

Figure 1. Model of adaptable mechanisms in V1. Desensitization can occur in any of three mechanisms: (1) an early stage (ES) that is untuned for orientation and is sensitive to a broad range of temporal frequencies; (2) a tuned mechanism that is sensitive to low temporal frequencies; (3) a normalization pool (NP) that is untuned for orientation and sensitive to a broad temporal frequency range overlapping with the range of the early stage. The early stage encompasses subcortical and input-layer cortical neurons, some of which (e.g., M-pathway neurons and neurons in the cortical input layers) are susceptible to adaptation $A_{\mathrm{ES}}$. The normalization stage pools across the output of early-stage and second-stage mechanisms, with desensitization occurring after pooling $A_{\mathrm{NP}}$. The output of the model neuron can also be influenced by adaptation, presumably through hyperpolarization $V_{\mathrm{t}}$.

frequency selective mechanism that linearly accumulates a selection of first-stage signals over its receptive field. The third is a divisive normalizing pool, also broadly tuned in space and time, which accumulates a selection of inputs from the first and second stages.

We consider the early stage as a pool of cells that are broadly tuned and consequently sensitive to uniform fields. These will mostly be cells in LGN and V1 input layers, but could also include cells in other cortical layers. Some (but not all) of the cells that contribute to the early stage are adaptable under the conditions tested here, namely, neurons in the magnocellular (M)-pathway and neurons in cortical input layers (Saul and Cynader, 1989b; Solomon et al., 2004). We assume that the input from the early stage to the second stage varies from one V1 neuron to the next, allowing for cell-by-cell variation in the fraction of adaptable input from the early stage. We further assume that the output of the second stage is regulated by a divisive normalizing signal. Because we used stimuli that were of optimal size, the component of the normalization signal which we are considering is that which operates within the classical receptive field. Suppression by mask stimuli is untuned for orientation (Morrone et al., 1982; Bonds, 1989; DeAngelis et al., 1992) and indicates that the normalization mechanism pools over a large number of first- and second-stage signals such that collectively, these inputs cover the full stimulus space. As normalization may already be present in subcortical neurons (Camp et al., 2009), the normalization pool of a cortical neuron may be sensitive to high temporal frequencies reflecting the high temporal sensitivity of geniculate neurons (Derrington and Lennie, 1984; Hawken et al., 1996). We depict this as an input from the early stage directly to the normalization pool (Fig. 1).

Each of the principal elements can be independently desensitized: the broadly tuned early stage, the output of the orientation-selective neuron, and the broadly tuned normalization pool. The early stage and the normalization pool both receive signals that ultimately derive from subcortical sources. However, these mechanisms may differ in their susceptibility to adaptation. For instance, the early stage inputs to the spatio-temporally bandpass second stage may draw entirely from nonadaptable (e.g., parvocellular) inputs while the normalization pool could sample more heavily from the M-cell component of the early stage.

In the model, the spike discharge of a spatio-temporally selective neuron arises after the membrane potential $(F(c))$ is rectified $\left(\bigsqcup_{+}\right)$scaled by a gain term $\left(S_{\text {spikes }}\right)$ and passed through a static nonlinearity $(\alpha)$. The tuning of adaptation effects has been explored in the orientation (Marlin et al., 1988; Hammond et al., 1989; Crowder et al., 2006), spatialfrequency (Movshon and Lennie, 1979; Albrecht et al., 1984; Saul and 
Cynader, 1989a), and temporal-frequency domains (Saul and Cynader, 1989b). Evidence accumulated across these studies suggests the tuned mechanism is desensitized when the adapting stimulus is well matched to the receptive field. We allow desensitization of the tuned mechanism to act via a hyperpolarizing $\left(V_{\mathrm{t}}\right)$ mechanism, as suggested by intracellular measurements (Carandini and Ferster, 1997; Sanchez-Vives et al., 2000a; Sanchez-Vives et al., 2000b), though not necessarily because the adapter evokes spikes (Sanchez-Vives et al., 2000a). Because of constraints imposed on other parameters, the range of $V_{\mathrm{t}}$ is restricted to lie between 0 and 1 (respectively no desensitization and full desensitization of the tuned mechanism, where full desensitization denotes hyperpolarization sufficient to conceal all membrane potential fluctuations below spike threshold) and thus is a measure of relative desensitization rather than an absolute measure of threshold in $\mathrm{mV}$.

$$
\begin{gathered}
R_{u}(c)=\left(S_{\text {spikes }} \cdot\left\lfloor F_{u}(c)\right\rfloor_{+}\right)^{\alpha} \\
R_{a}(c)=\left(S_{\text {spikes }} \cdot\left\lfloor F_{a}(c)-V_{t}\right\rfloor_{+}\right)^{\alpha} .
\end{gathered}
$$

The membrane potential of the spatio-temporally selective mechanism is constructed by dividing the early stage by the normalizing signal.

$$
\begin{gathered}
F_{u}(c)=\frac{G \cdot \mathrm{c}}{\sqrt{\sigma^{2}+(\mathrm{c})^{2}}} \\
F_{a}(c)=\frac{A_{\mathrm{ES}} \cdot G \cdot \mathrm{c}}{\sqrt{\sigma^{2}+\left(A_{\mathrm{NP}} \cdot c\right)^{2}}} .
\end{gathered}
$$

Here, $c$ is the input contrast. The constant $G$ is a gain term to scale the linear response. The constant $\sigma$ represents the semisaturation contrast of the generator potential and relates to the strength of the normalizing signal. In general, the membrane potential also depends on the projection of the stimulus onto the linear mechanism's receptive field. However, because we measured the contrast response only using optimal drifting gratings we need only consider contrast here (Tailby et al., 2008). The coefficient $A_{\mathrm{ES}}$ represents the extent to which the set of early stage signals drawn upon by the second stage are desensitized; the coefficient $A_{\mathrm{NP}}$ represents the extent to which the normalization pool is desensitized (with this single parameter subsuming desensitization in both its earlystage and second-stage inputs). We assume that adaptation can only reduce the signal amplitude transmitted through the early stage and normalization pool, so $A_{\mathrm{ES}}$ and $A_{\mathrm{NP}}$ are bounded by 0 (full desensitization) and 1 (no desensitization).

We fit the above model concurrently to the responses collected in the control conditions and any adaptation condition by least-squares minimization. We forced the gain terms, the nonlinearity and the semisaturation contrast to have the same values across all conditions. For reasons described below (see Results, Isolation of adaptable mechanisms), adaptation effects on the early stage were constrained to be identical for adaptation conditions that were matched in temporal frequency, irrespective of adapter orientation. We imposed this same constraint on the desensitization of the normalization pool. We allowed the spatiotemporally tuned mechanism to be desensitized only by stimuli that were well matched to the receptive field. When all three mechanisms can be desensitized, the model is under-constrained. However, we can address this problem by characterizing a neuron in adaptation conditions that are matched in adapter temporal frequency, but differ in their ability to desensitize the tuned mechanism.

Expression of adaptation. Our model characterizes mechanisms underlying adaptation effects in V1 neurons, but we would like also to understand how those adaptation effects are expressed in attributes of the contrast-response function: contrast gain (a measure of the neuron's contrast sensitivity) and response gain (the neuron's input/output transformation, represented by the slope of the curve). A neuron's contrast gain determines the potentially distinguishable range of contrasts; the response gain controls how different are the responses to those contrasts.

Contrast response functions of V1 neurons in cat and primate are well described by a hyperbolic ratio function (Albrecht and Hamilton, 1982). When contrast response functions saturate in both the control and adaptation conditions, changes in contrast gain and response gain can be extracted

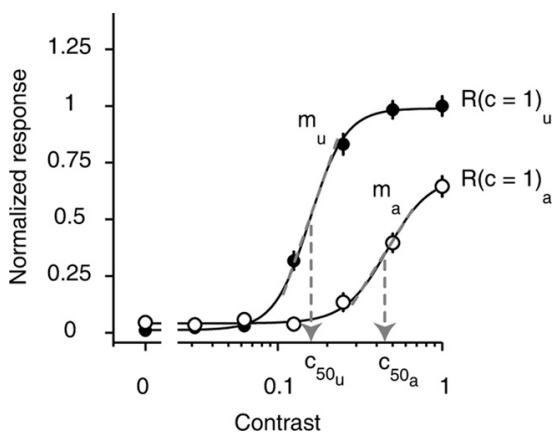

Figure 2. Computation of gain changes following adaptation. CGAl is computed as the ratio of the contrasts that produce half the maximum response. RGAl is computed as the ratio of the best fitting linear slopes through the rising portion of the contrast response when plotted on logarithmic abscissae. MRAl is computed as the ratio of the responses to full contrast stimuli. Data from cell of Figure $3 B$, top row, for low temporal frequency preferred adapter. Smooth lines are best-fitting hyperbolic ratio functions.

from the relevant parameters of the hyperbolic ratio function (Albrecht et al., 1984; Sclar et al., 1989). In our data contrast response functions were often not saturated (particularly under adaptation conditions), and estimates of contrast gain and response gain were poorly constrained. We therefore require a more robust means of characterizing the changes in contrast gain and response gain brought about by adaptation.

To characterize the change in contrast gain, we follow Movshon and Lennie (1979) and compute (from fits of the hyperbolic ratio function) the ratio of contrasts that generate half the maximum response in the control and adaptation conditions (Fig. 2). We call this ratio the Contrast Gain Adaptation Index (CGAI):

$$
\text { CGAI }=\frac{c_{50 u}}{c_{50 a}},
$$

where $c_{50_{a}}$ is the contrast that elicits half the response to full contrast in the adaptation condition and $c_{50_{u}}$ is the contrast that elicits half the response to full contrast in the control condition.

To characterize changes in response gain, we compute the ratio of the slope (as a function of log contrast) of contrast response when the cell is in the control condition to the slope when the cell is in the adaptation condition (Fig. 2). This is the Response Gain Adaptation Index (RGAI):

$$
\mathrm{RGAI}=\frac{m_{a}}{m_{u}},
$$

where $m$ is the slope of a best fitting line to the linear portion of the contrast response function plotted on a logarithmic abscissa. We identify the linear portion of the fitted curve as the region bounded by the contrasts that produced maximum acceleration and deceleration in the response. If there was no saturation, and thus no deceleration, the upper bound of the contrast range was set to full contrast.

Among neurons for which the hyperbolic ratio function yielded well constrained fits to contrast-response curves, the estimates of change in contrast gain and response gain from the fitted parameters were highly correlated with CGAI and RGAI ( $r=0.96$ and 0.73 , respectively). In what follows we use CGAI and RGAI to represent contrast gain and response gain.

Some physiological experiments (Maffei et al., 1973; Vautin and Berkley, 1977; Albrecht et al., 1984) have characterized contrast adaptation effects by the decline in the response to the high-contrast adapter. We can capture this aspect of contrast adaptation by comparing the magnitude of the response at full contrast in the control and adaptation conditions (Fig. 2). We compute the Maximum Response Adaptation Index (MRAI) as:

$$
\text { MRAI }=\frac{R(c=1)_{a}}{R(c=1)_{u}} .
$$

Because a change in MRAI can result from a change in either response gain or contrast gain (when the curve is not saturated) it is an ambiguous 
measure of the expression of adaptation. We compute it to permit comparison of our results with those from earlier studies.

In what follows we use these three metrics-CGAI, RGAI and MRAI - to characterize the changes brought about by adaptation. Values less than one indicate an adaptation-induced loss of contrast gain, response gain, or response at full contrast, respectively. Values greater than one indicate an increase in contrast gain, in response gain or in the response at full contrast.

To determine the significance of changes in contrast gain and response gain, we used a bootstrap analysis in the manner of Solomon et al. (2004). For each test contrast presented, we drew $N$ random samples with replacement (typically 10 for the adaptation condition and 20 for the control condition) from the array of responses and computed the adaptation indices. We repeated this procedure 1000 times. From this array we computed the means, SDs and confidence intervals of the changes caused by adaptation.

\section{Results}

We report here on responses from 91 neurons recorded in primary visual cortex. Simple and complex cells were distinguished by the ratio of the firing rate modulation at the stimulus frequency $(\mathrm{F} 1)$ to the mean discharge rate (F0) in response to a full-contrast, spatio-temporally optimal drifting grating (Skottun et al., 1991). If F1 was greater than or equal to F0, the cell was classified as simple, otherwise as complex. In the analysis that follows, the response of a simple cell is the magnitude of $\mathrm{F} 1$; the response of a complex cell is the average discharge rate after subtracting the spontaneous rate. Simple and complex cells did not differ in their responses to the stimulus manipulations described here, so are not discussed separately.

\section{Isolation of adaptable mechanisms}

We revealed distinct adaptable mechanisms by appropriate choice of the spatial and temporal parameters of adapting gratings. In our model a cortical neuron receives-in addition to unadaptable inputs - an adaptable input from a first stage comprised of neurons in the M-pathway and neurons in the cortical input layers. Neurons in the M-pathway lack orientation selectivity and are adapted only by stimuli modulated at high temporal frequencies (Solomon et al., 2004). Neurons in the cortical input layers have poor orientation tuning (Hawken and Parker, 1984; Gur et al., 2005) and are adapted also by more slowly modulated stimuli (Saul and Cynader, 1989b). Therefore the adaptable early stage is broadly tuned in orientation and temporal frequency; it is modeled here as a single stage though it potentially comprises multiple neural substrates, both cortical and subcortical. Stage two consists of orientation-selective neurons in V1 that are sensitive to (and adapted by) low temporal frequencies (and insensitive to the high temporal frequencies that drive the first stage well). Stage three is the normalization pool, taken here to primarily reflect a suppressive mechanism that is coextensive with the classical receptive field. Response suppression is untuned for orientation and low-pass for spatial frequency (Morrone et al., 1982; Bonds, 1989; DeAngelis et al., 1992). We therefore model the normalization pool as an untuned mechanism which draws inputs from broadly tuned early-stage signals and from orientation-selective cortical neurons tuned to all orientations. We presume that the normalization pool is sensitive to a broad temporal frequency range reflecting the overall sensitivity profile of the neurons on which it draws, an assumption supported by measurements of the tuning of gain-control mechanisms in cat (Freeman et al., 2002). Some of its sensitivity to high temporal frequencies may be inherited from normalization in subcortical inputs (Camp et al., 2009). Differences between the effectiveness of adapters that desensitize the distinct subcortical and cortical components of the early stage and the normalization pool are absorbed into the parameter values $A_{\mathrm{ES}}$ and $A_{\mathrm{NP}}$.

We deployed up to four types of adapters intended to isolate the presumed different adaptable mechanisms. Specifically, we used full contrast stimuli modulated at high rates $(30-50 \mathrm{~Hz})$ to avoid desensitizing the tuned mechanism and at low rates (1-2 $\mathrm{Hz}$ ) to minimize desensitization of the subcortical components of the early stage and the normalization pool. At each temporal frequency we used adapters at two orientations, parallel with and orthogonal to the cell's preferred orientation (termed "preferred" and "null," respectively). Because cells in the LGN lack orientation selectivity, we expect comparable adaptation from the two high temporal frequency adapters. Conversely, because most cortical neurons are strongly orientation-selective and past studies have suggested that adaptation effects are tuned, we expect low temporal frequency adapters at the preferred orientation to desensitize cortical cells more than will low temporal frequency adapters at the null orientation because these act upon the spatiotemporally tuned mechanism.

Most neurons in our population were strongly orientation tuned and had high temporal frequency cutoffs below $30 \mathrm{~Hz}$. Consequently, few responded to any but the low temporal frequency preferred adapter. If one of the other three adapters did evoke a significant response from a cell $(>10 \%$ maximum unadapted response), we excluded the cell from that condition. By this criterion, we excluded 5 high temporal frequency preferred and 10 low temporal frequency null adapter conditions (of 46 and 60 , respectively).

Figure 3 shows the effects of the different adaptation conditions on four cells in V1 that represent the diversity of effects observed. Figure $3 A$ shows each neurons' contrast response before and after adaptation to a full contrast grating drifting at 30 $\mathrm{Hz}$, presented at each cell's preferred orientation (open circles) or null orientation (open triangles). Figure $3 B$ shows corresponding measurements in the same cells for low temporal frequency adapters presented at each cell's preferred orientation (open diamonds) or null orientation (open squares). Control measurements (filled circles) are identical in Figure 3, $A$ and $B$.

Adaptation effects induced by high temporal frequency gratings were not orientation-specific, suggesting desensitization of untuned mechanisms. High temporal frequency adaptation usually shifted the contrast response functions rightward on the semilogarithmic plot (Fig. $3 A$, top three rows), even though the adapter evoked no response. For the neurons in the first and third rows (Fig. 3A), and many others, adaptation led to steeper contrast response functions, and in some cases (Fig. $3 A$, top row) response facilitation at high contrasts. However, these changes were not always coupled: adaptation could bring about rightward shifts of the contrast response function without any change in the maximum response or the steepness of the curve (Fig. $3 A$, second row) or it could change the steepness of the curve without changing its horizontal position (Fig. $3 A$, bottom row).

Adaptation effects caused by low temporal frequency gratings at both preferred and null orientations brought about substantial, though different, changes in contrast response functions (Fig. $3 B$ ). The preferred adapter caused a large rightward shift in the curves, a reduction of the maximum response and a change in the steepness of the curve (open diamonds). The null adapter (open squares), which itself evoked no response, usually shifted the contrast response function rightward (Fig. 3B, top, second, and bottom rows), though not always (Fig. $3 B$, third row). The rightward shift induced by null adapters was almost always less 
A
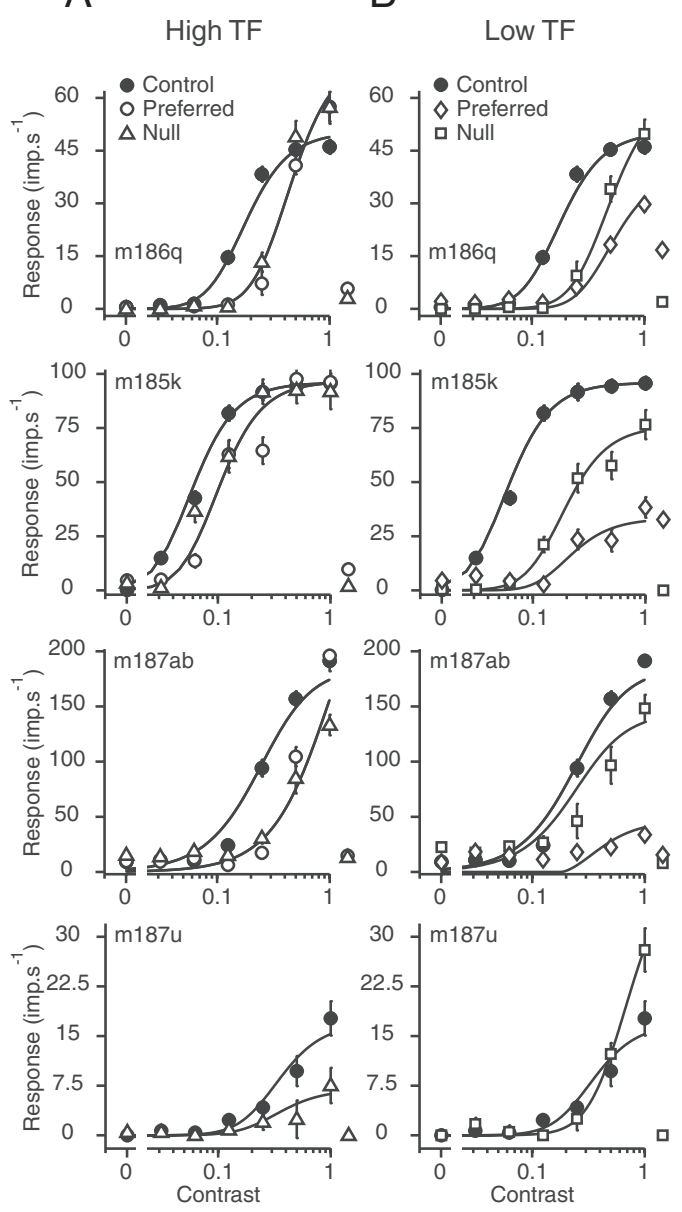

Figure 3. Changes in response brought about by adaptation to different stimuli. $A$, Contrast response functions of four cells when the cell was unadapted (filled symbols) or adapted to a high temporal frequency grating at full-contrast (open symbols), at the preferred orientation (circles) or null orientation (triangles). $\boldsymbol{B}$, Contrast response functions of the same four cells when the cell was unadapted (filled symbols; same as in $\boldsymbol{A}$ ) or adapted to a low temporal frequency grating at full contrast (open symbols) at the preferred orientation (diamonds) or null orientation (squares). Smooth lines are best fitting solutions to the model. Responses to the different adapters (averages over the first second of adaptation) are plotted to the right of each panel with the appropriate symbol. The response to the adapter could be different from the response to the full contrast stimulus in the low temporal frequency preferred adaptation condition because the adapting stimulus was modulated at a lower temporal frequency (usually $1-2 \mathrm{~Hz}$ ) than the optimal temporal frequency probes. Vertical bars denote \pm 1 SEM. Example cell in bottom row was characterized for adaptation only to stimuli at the null orientation.

than that induced by preferred adapters. The adaptable mechanism in the M-pathway is not sensitive to the low temporal frequencies used here (Solomon et al., 2004). That there was a rightward shift at all, with reduced response to low contrast probes, suggests that the early stage is comprised of two components-one derived from M-cells and another from nonoriented neurons early in visual cortex that are sensitive to low temporal frequencies.

Often a neuron was susceptible to contrast adaptation by both high temporal frequency stimuli (at any orientation) and low temporal frequency stimuli at the null orientation. Sometimes these stimuli had similar effects (Fig. 3, top row), but not always (Fig. 3, second through bottom rows). Responses could be reduced following adaptation in one temporal frequency condition but sensitized after another, even though neither adapter evoked a response from the cell (Fig. 3, bottom row). This is a second
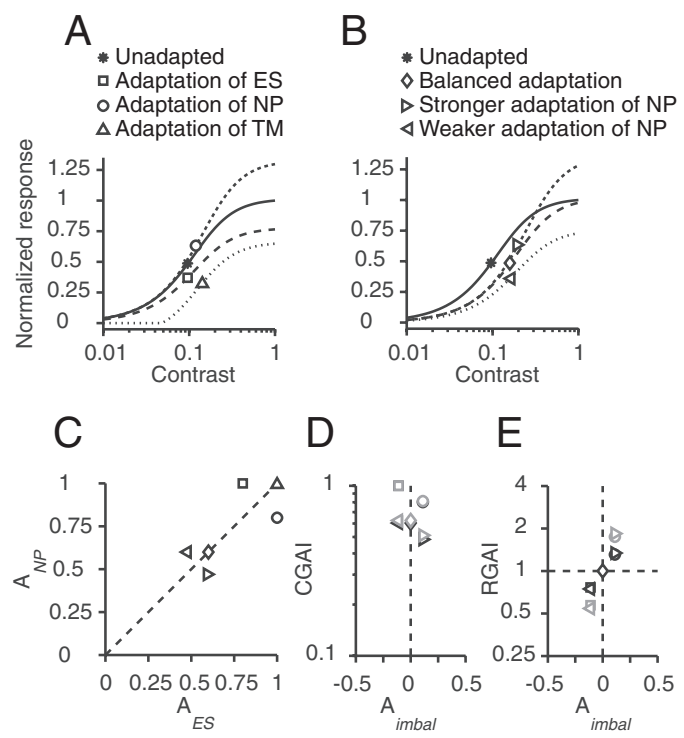

Figure 4. How desensitization of different mechanisms affects a cell's response. $A$, Contrast response functions before and after adaptation occurring separately in each adaptable mechanism. The solid line shows the control condition contrast response. The long dashed line shows the effect of desensitizing only the early stage. The short dashed line shows the effect of desensitizing only the normalization pool. The dotted line shows the effect of desensitizing only the spatio-temporally tuned mechanism. $\boldsymbol{B}$, Contrast response functions before and after the early stage and the normalization pool are desensitized concurrently. The solid line is the control condition contrast response curve (same as in $\boldsymbol{A}$ ). The long dashed line shows the effect of desensitizing the two mechanisms equally. The short dashed line shows the effect of desensitizing the normalization pool more than the early stage. The dotted line shows the effect of desensitizing the early stage more than the normalization pool. Symbols in $\boldsymbol{A}$ and $\boldsymbol{B}$ are plotted at the semisaturation point of the corresponding contrast response function. $C$, Values of the adaptation parameters $\left(A_{\mathrm{ES}}\right.$ and $\left.A_{\mathrm{NP}}\right)$ that produced the contrast response functions in $\boldsymbol{A}$ and $\boldsymbol{B}$. $\boldsymbol{D}, \boldsymbol{E}$, Effect of changing the exponent $(\alpha)$ on the expression of adaptation through CGAI (D) and RGAI $(\boldsymbol{E})$. Black symbols represent gain change for the exponent value used in $\boldsymbol{A}$ and $\boldsymbol{B}$; gray symbols represent gain change for an exponent twice as large (curves not shown in $\boldsymbol{A}$ and $\boldsymbol{B}$ ). Symbols in $\boldsymbol{D}$ and $\boldsymbol{E}$ correspond to the adaptation parameters shown in $\boldsymbol{C}$.

indication that the early stage is made up of multiple parts and furthermore that there are distinct untuned mechanisms (the early stage and the normalization pool).

The smooth lines in Figure 3 show best fits of our model (Eqs. 1,2). In Figure $3 A$ high temporal frequency adapters at both orientations produce the same curve because we forced both the early stage and the normalization pool to be insensitive to variations of adapter orientation and the tuned mechanism to be insensitive to the high temporal frequency adapter. In Figure $3 B$ the differences between the adaptation curves are attributable entirely to desensitization of the spatio-temporally tuned mechanism. The model explained on average $94 \%$ of the variance in the data. We excluded $\sim 5 \%$ of the conditions run because the model fits returned values at the parameter bounds. Including these conditions changed neither the significance nor tenor of the results.

\section{Model behavior}

The effects of adaptation on the different mechanisms act in combination to bring about the complex changes in the behavior of neurons seen in Figure 3. Figure 4 illustrates how these component mechanisms, acting alone and in combination, alter the contrast-response curve of a hypothetical neuron. Desensitization of the broadly tuned early stage alone reduces the response at every contrast (Fig. $4 A$, long dashes). This behavior is seen in the cells in Figure 3 (Fig. 3A, bottom row; $3 B$, third row). Conversely, 
desensitization of the normalization pool alone (Fig. 4A, short dashes) results in increased responses. Desensitization of either mechanism in isolation changes the response gain. Desensitization of the early stage does not affect the horizontal position of the contrast response function; desensitization of the normalization pool, by extending the operating range of the cell, shifts the contrast response function rightward (the semisaturation contrast of each curve is highlighted by the symbol locations in Fig. $4 A, B$ ).

If the signals from both the early stage and the normalization pool are equally desensitized by adaptation, the contrast response function shifts horizontally, resulting in a pure contrast gain change (as if the input contrast were reduced by a multiplicative factor: Fig. $4 B$ long dashes and cell depicted in Fig. $3 A$, second row). Response gain change can arise only if the early stage and the normalization pool are differentially desensitized. When the early stage is more desensitized than is the normalization pool, contrast gain and response gain are both reduced (Figs. $4 B$, dotted line; $3 B$, second row, open squares). This resembles the effect of isolating desensitization to the spatio-temporally tuned mechanism (Fig. $4 A$ dotted line). However, if the normalization pool is desensitized more than the early stage, the contrast response function is shifted horizontally (contrast gain is reduced) but response gain is paradoxically increased (Figs. $4 B$, short dashed line; $3 A$, top row; $3 B$, top and bottom rows). This can result in an increased response at high contrasts (Figs. $4 B$, short dashed line; $3 A$, top row; $3 B$, bottom row) - something we observed often.

Figure $4 C$ shows the amounts by which the early stage and the normalization pool are desensitized to produce the contrast response functions in the simulations (for a particular choice of exponent and contrast sensitivity). The gain changes brought about by desensitization of the different adaptable mechanisms map to different points in this parameter space. One can then use the parameter space for a given cell to learn how adaptation may be expressed in terms of contrast gain and response gain. Equal desensitization of the early stage and the normalization pool plots along the unit diagonal and signifies a pure contrast gain change. Points that lie off the line reflect an imbalance in the desensitization of the early stage and the normalization pool and indicate a response gain change. We quantify the imbalance in adaptation effects on the early stage and the normalization pool by computing the ratio of their difference divided by their sum $\left(A_{\text {imbal }}=\right.$ $\left.\left(A_{\mathrm{ES}}-A_{\mathrm{NP}}\right) /\left(A_{\mathrm{ES}}+A_{\mathrm{NP}}\right)\right)$. The sign of the ratio tells us the direction of the change in response gain. Positive values; which correspond to points below the diagonal, signify greater desensitization of the normalization pool and consequently an increase in response gain.

Inspection of Figure $4, A$ and $B$ (see also Predicting gain change from desensitization of underlying mechanisms, below), indicates that desensitization of the normalization pool, but not the early stage, is sufficient to bring about a contrast gain change. Therefore, points closer to the abscissa represent greater reductions of contrast gain. Desensitization of the tuned mechanism always acts to reduce contrast gain and response gain (Fig. 4A). Its presence confounds the prediction of gain change arising from desensitization of the nonoriented mechanisms. If desensitization of the normalization pool is accompanied by desensitization of the spatio-temporally tuned mechanism the net effect can be a reduction in response gain, because adaption of the tuned mechanism undoes the increase in response gain otherwise brought about by reducing the output of the normalization pool (Fig. $3 B$, top row, open diamonds).

The exponent term $(\alpha)$ and the semisaturation contrast $(\sigma)$, which respectively capture the baseline steepness and the sensi- tivity of the contrast response (Eqs. 1,2), are not represented in Figure $4 C$. Because both of these parameters can differ from cell to cell, it is important to know whether their values affect estimates of gain change. If not, then knowing the extent to which each underlying mechanism is desensitized will be sufficient to characterize the gain change. We simulated two families of contrast response functions with identical adaptation parameters shown in Figure 4C. The families differed in their exponent term, one with the value used in Figure 4, $A$ and $B$, and another twice as steep (curves not shown). This manipulation affected estimates of CGAI by a small amount (Fig. $4 D$, similarity of matched black and gray symbols). On the other hand, the value of the exponent greatly affected estimates of RGAI (Fig. $4 E$ ). A similar manipulation of the semisaturation contrast (one with the value used in Fig. $4 A, B$ and another twice as large) indicated that cell-to-cell variation in semisaturation contrast has a small effect on estimates of CGAI and no effect on estimates of RGAI (data not shown). The effect that the underlying contrast sensitivity and the exponent term have on estimates of CGAI occurs when the adaptation acts to shift the contrast response function (usually in the adapted condition) rightward so profoundly that saturation is lost. For such cells estimates of CGAI will be too large (because the estimate of $c_{50_{a}}$ is underestimated; see Predicting gain change from desensitization of underlying mechanisms, below).

\section{Desensitization of underlying mechanisms}

Figure 5, $A$ and $B$, shows how the early stage and the normalization pool were desensitized by adaptation to stimuli modulated at high temporal frequencies (Fig. 5A) and low temporal frequencies (Fig. $5 B$ ) across our population. Points lie below the diagonal when the normalization pool is desensitized more than the early stage. The two mechanisms were often desensitized to a similar degree (most points lie close to the unit diagonal). Nevertheless, the small imbalances account for much of the diversity in the effects of adaptation expressed in terms of gain change (see preceding section).

At low temporal frequencies the tuned mechanism is desensitized by adaptation to gratings at the preferred orientation. The scatter plot in Figure $5 C$ shows this as a function of the imbalance ratio. $A_{\mathrm{ES}}$ and $A_{\mathrm{NP}}$ differ only across temporal frequency, never for orientation. Thus for Figure $5 A$, if a cell was characterized in either high temporal frequency condition (or both) it appears as a single point in this plot. Because $V_{\mathrm{t}}$ can trade off against the other model parameters $\left(A_{\mathrm{ES}}\right.$ and $A_{\mathrm{NP}}$, shown graphically by dotted lines in Fig. $4 A, B$ ), Figure 5, $B$ and $C$, includes only those cells for which fits were adequately constrained by measurement in the low temporal frequency orthogonal condition. The amount the tuned mechanism was desensitized was largely independent of the absolute or the relative desensitization of the other two mechanisms. Because the tuned mechanism was not allowed to be affected by the high temporal frequency adapters, the position of points relative to the diagonal in Figure $5 A$ represent the direction of response gain change unambiguously.

The cells most susceptible to desensitization of the early stage in the high temporal frequency conditions were also those with the greatest contrast sensitivity (lowest $\sigma ; r=0.43, p<0.05$ ). These cells also showed the greatest surround suppression, estimated from initial measurements of size-tuning $(r=0.25, p<$ $0.05)$. There was a hint that these cells also were sensitive to higher temporal frequencies, although this trend did not reach statistical significance $(r=0.18 ; p=0.12)$. We comment in the Discussion on the relationship between desensitization of the early stage mechanism and the descriptive tuning parameters. 


\section{Effects of contrast adaptation on contrast gain, response gain and maximum response}

The complex interplay of the effects of adaptation through multiple mechanisms results in overall changes in contrast gain and response gain that can be simply captured by metrics derived directly from measurements. Figure 6 shows RGAI plotted against CGAI for each of our adapting conditions. Vertical and horizontal dotted lines indicate no change in CGAI and RGAI, respectively. Points to the left or below the lines represent a loss of contrast gain or response gain, respectively. Low temporal frequency preferred adapters reduced contrast gain in nearly every cell (Fig. $6 A)$. Low temporal frequency null adapters (to which the neurons did not respond) also reduced the contrast gain of many cells (Fig. $6 B)$, although by less than in the preferred condition. Comparing the CGAI in the two low temporal frequency conditions shows that an adapter at the preferred orientation almost always induces greater loss of contrast gain (Fig. 6C) $(p<0.01)$.

High temporal frequency adapters often reduced contrast gain, about equally for both orientations of adapter (Fig. $6 D, E)$. The CGAI is strongly correlated for the two high temporal frequency adaptation conditions $(r=0.73 ; p<0.01)$ as would be expected if the adaptation effects are expressed only through untuned mechanisms (Fig. 6F). Approximately $39 \%$ of the cells, dispersed throughout the cortical laminae, exhibited significant contrast gain reductions during the two high temporal frequency conditions.

Different adapters affected response gain quite differently. Low temporal frequency preferred adapters reduced response gain in some cells, but increased it in as many others, and the changes in response gain were unrelated to the changes in contrast gain (Fig. 6A). For the other three adapting conditions (Fig. 6B, D,E), cells tended to cluster in the upper left quadrant, indicating that adaptation increased response gain as it decreased contrast gain. In these conditions, contrast response functions most commonly shifted rightward and became steeper following adaptation. In the context of the model presented here, this indicates stronger desensitization of the normalization pool than of the early stage (with the tuned mechanism, by definition, being unaffected by these three conditions). Similar to the effects on contrast gain, we found that the RGAI measured in the two high temporal frequency conditions was correlated $(r=0.41 ; p<0.05)$.

Figure 7 shows histograms of the MRAI for the four adapting conditions used. Low temporal frequency preferred adapters reduced the maximum response in almost every cell. On average, low temporal frequency preferred adapters reduced the maximum reresponse.

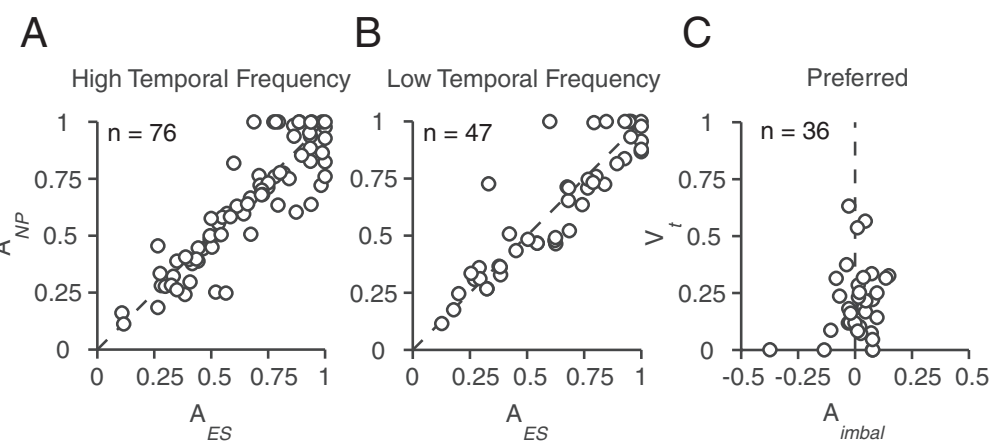

Figure 5. $\boldsymbol{A}, \boldsymbol{B}$, Extent to which the early stage $\left(A_{\mathrm{ES}}\right)$ and the normalization pool $\left(A_{\mathrm{NP}}\right)$ are desensitized by high temporal frequency adapters $(\boldsymbol{A})$ and by low temporal frequency adapters $(\boldsymbol{B})$. Points along the diagonal represent cells in which the two mechanisms are desensitized equally. Points below the diagonal denote cells with stronger desensitization of the normalization pool, resulting in an increased response to high contrast stimuli. $C$, How desensitization of the tuned mechanism varies with the ratio of the desensitization in the early stage and normalization pool. Points with values greater than zero indicate a decrease in
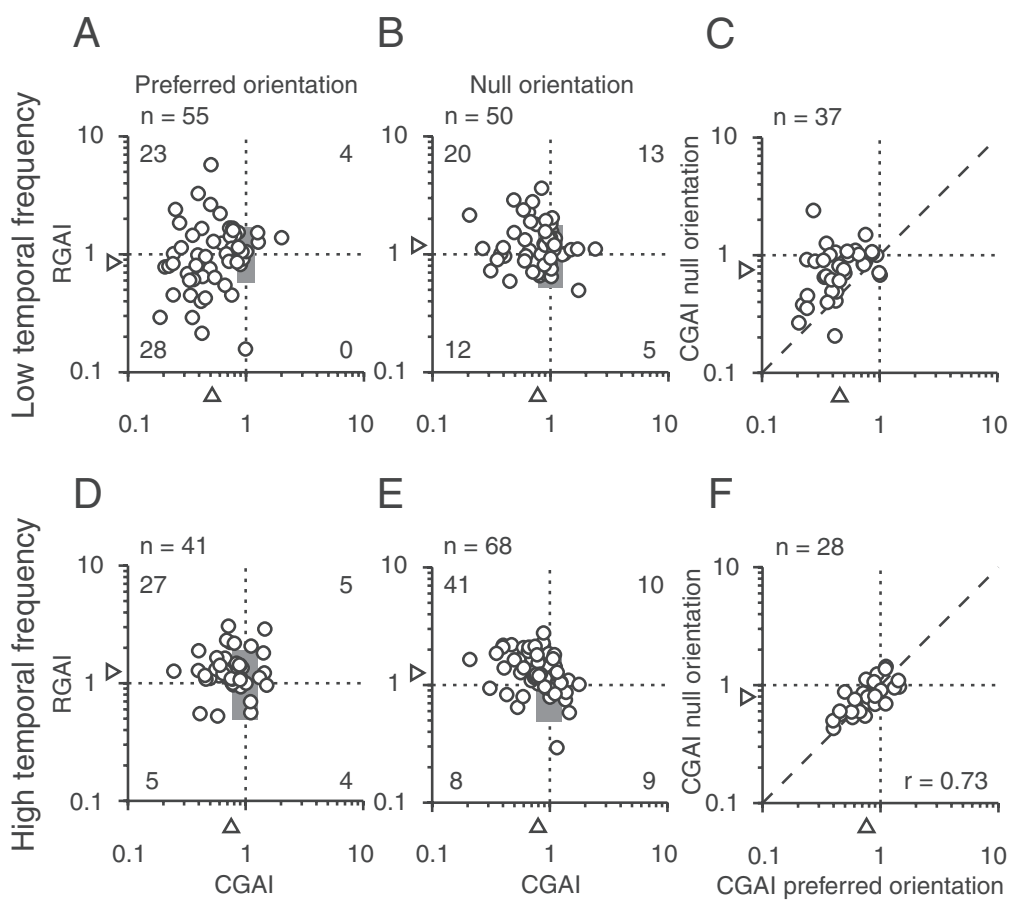

Figure 6. Effects of different adapting conditions on contrast gain (CGAl) and response gain (RGAl). $A$, Low temporal frequency adapter at the preferred orientation; $\boldsymbol{B}$, low temporal frequency adapter at the null orientation; $\boldsymbol{D}$, high temporal frequency adapter at the preferred orientation; and $\boldsymbol{E}$, high temporal frequency adapter at the null orientation. Vertical and horizontal dotted lines denote no change in CGAI and RGAl, respectively. Triangles on the axes show means. Gray boxes at the center of each plot indicate average $95 \%$ confidence intervals derived from a bootstrap procedure. Points outside the rectangles identify cells that are significantly affected by adaptation. The number of cells in each quadrant is indicated by the number in the corner. $\boldsymbol{C}, \boldsymbol{F}, \mathbf{C G A I}$ for the two low temporal frequency $(\boldsymbol{C})$ and high temporal frequency $(\boldsymbol{F})$ adapters.

sponse to the optimal grating to $38 \%$ of the unadapted response, an amount similar to that found by Albrecht et al. (1984). However, adaptation did cause an increase in maximum response for a small number of neurons, consistent with Sclar et al. (1989). The other three adapting conditions had little effect on maximum response.

\section{Predicting gain change from desensitization of underlying mechanisms}

Figure 5 tells us about the weights with which the adaptable mechanisms contribute to the overall effects of adaptation for a cell. Figure 6 tells us about the gain changes brought about by adaptation. We want to understand how well differential desen- 
A

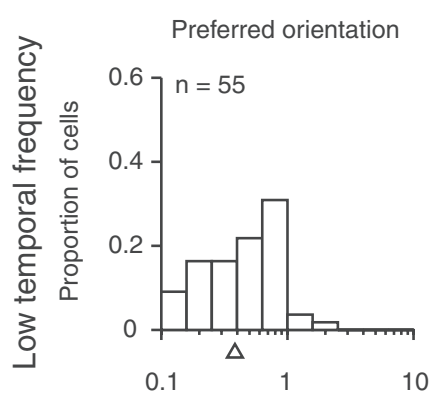

B

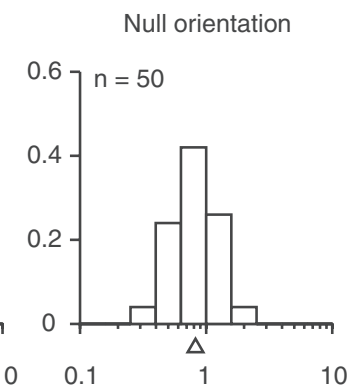

C

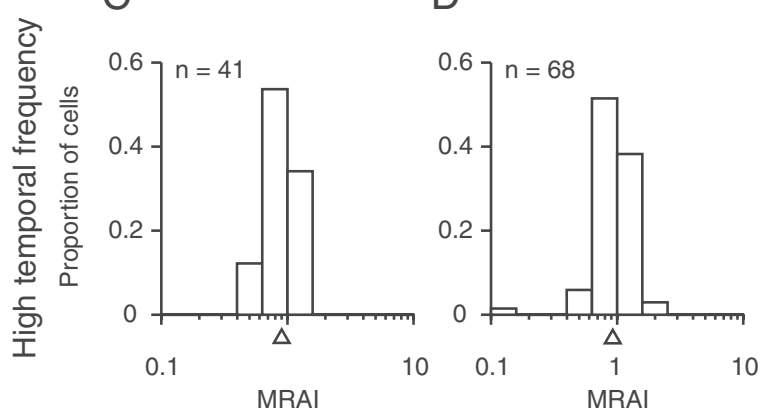

Figure 7. Effect of adaptation on maximum response. Panels show distribution of MRAI following adaptation under four different conditions: low temporal frequency gratings at the preferred orientation $(\boldsymbol{A})$, low temporal frequency gratings at the null orientation $(\boldsymbol{B})$, high temporal frequency gratings at the preferred orientation $(\boldsymbol{C})$, and high temporal frequency gratings at the null orientation $(\boldsymbol{D})$. Triangles on the axes show means.

A Low TF; null orientation
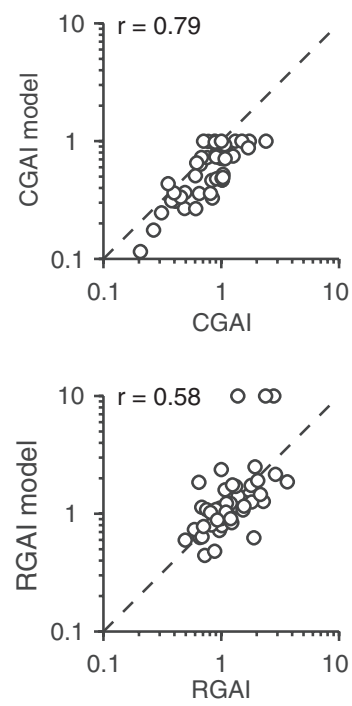

\section{B}

High TF; preferred orientation
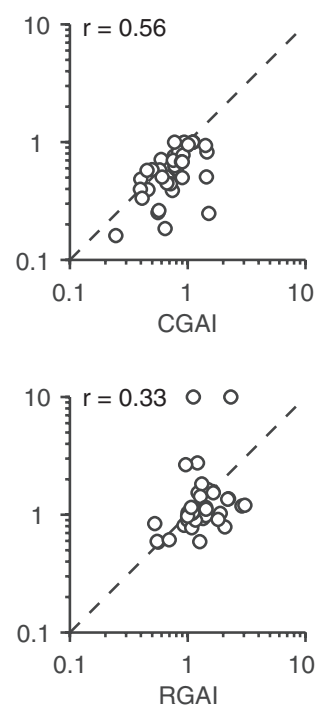

C

High TF; null orientation
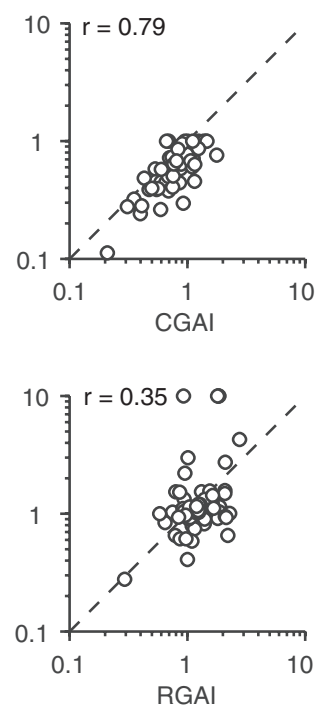

Figure 8. Comparison of gain changes estimated from the model and those derived from data. CGAl (top row) and RGAI (bottom row) values estimated from the model are plotted against those derived from the data for low temporal frequency gratings at the null orientation $(\boldsymbol{A})$, high temporal frequency gratings at the preferred orientation $(\boldsymbol{B})$, and high temporal frequency gratings at the null orientation (C). Correlation coefficients (all significant $p<0.05$ ) are shown in top left corner of each panel.

sitization of the underlying mechanisms accounts for the full diversity of gain changes expressed by V1 neurons. We can explore the expository utility of the model by asking how well we can predict changes in the descriptive measures, CGAI and RGAI, from model estimates of how much the underlying mechanisms are desensitized.

In the special case where there is no desensitization of the spatio-temporally tuned mechanism $\left(V_{\mathrm{t}}=0\right)$ we can rearrange Equations 1 and 2 to estimate the gain change metrics as:

$$
\begin{gathered}
\mathrm{CGAI}_{\text {model }}=A_{\mathrm{NP}} \\
\mathrm{RGAI}_{\text {model }}=\left(\frac{A_{\mathrm{ES}}}{A_{\mathrm{NP}}}\right)^{\alpha} .
\end{gathered}
$$

From the above equations, we see that changes in contrast gain depend only on the amount that the normalization pool is desensitized by the adapter. The response gain change depends on the ratio of the desensitization of the two untuned mechanisms as well as the exponent term $(\alpha)$.

Figure 8 shows the relationship between observed values of CGAI and those predicted by the model, for the three conditions that isolate the untuned adaptable mechanisms. The two measures of contrast gain are highly correlated in all three conditions, though estimates of the change in contrast gain caused by adaptation from the model are larger than estimates from the data (points lay below the unity line). The small discrepancy between the two estimates of adaptation-induced contrast gain change is due to those cells lacking saturation. Often the adaptation effects were so profound that the saturating portion of the contrast response function was not fully captured in the adapted condition. This introduces a small bias in the estimate of $c_{50}$-it is lower than would have been expected were stimuli of $>100 \%$ contrast physically realizable. When carried through the computation of CGAI (Eq. 3), nonsaturated curves will tend to cause an underestimation in the amount of contrast gain change- the CGAI values are too large. This problem is exacerbated when the contrast response function is steep but still not saturated. On the other hand, our multiple mechanisms model parameter-driven estimates of the change in contrast gain brought about by adaptation extrapolate beyond $100 \%$ contrast based on the contrast responses collected under all conditions, including the unadapted condition. Indeed differences between the two estimates of contrast gain change are negligible when we allow nonphysical contrasts $(>100 \%)$ under simulation (data not shown). We note that the discrepancy between the two estimates in any case is small.

Similarly for response gain, the estimates from the model and the data were significantly correlated. However, the model predicted response gain change less well than it did contrast gain change. We note that changes in response gain are inherently more difficult to characterize as the average confidence interval for RGAI is much larger than that for CGAI (Fig. 6 - the gray boxes are vertical rectangles, not squares). Furthermore, model predictions of RGAI depend on three parameters from the model, rather than one for CGAI. Errors in estimating multiple parameters accumulate in the com- 
putation of RGAI from the model. The greater difficulty in estimating RGAI, from both the data and the model, likely explain their poorer correlation (relative to CGAI). In the case of low temporal frequency preferred orientation adapting condition (data not shown), estimates of gain change from the model did not match those derived from the data, as expected because desensitization of the tuned mechanism also contributes to the effects on gain following adaptation.

\section{Discussion}

\section{Multiple adaptable mechanisms}

Prolonged exposure to high temporal frequency and low temporal frequency stimuli induces changes in the shape of the contrast response function of V1 neurons. However the effects of adaptation to these stimuli are expressed in different ways and suggest desensitization of different mechanisms. Effects of adaptation brought about by high temporal frequency stimuli are untuned for orientation (Fig. $6 F$ ) and probably arise in the M-pathway (Solomon et al., 2004). Orientation-selective effects of adaptation brought about by low temporal frequency stimuli must originate in cortex. This has been characterized in previous studies (Maffei et al., 1973; Ohzawa et al., 1985). Although effects of adaptation to low temporal frequency stimuli are tuned, a high contrast grating presented at the null orientation-to which the cell does not respond-induces adaptation that implicates an untuned input to most V1 neurons that is excitatory, subthreshold and adaptable. This phenomenon was noted in earlier work (Ohzawa et al., 1985) and is consistent with a recent study of adaptation in cat V1 (Crowder et al., 2006).

The effect of low temporal frequency adapters at the null orientation is greater than could be conveyed by the very weak adaptation that is sometimes observed in parvocellular neurons (Solomon et al., 2004; Tailby et al., 2008; Camp et al., 2009). This adaptable untuned input probably arises early in V1, where many input layer neurons are broadly orientation tuned or nonoriented, though it could, in principle, comprise neuronal pools in other laminae. Priebe and Ferster (2006) and Tailby et al. (2008) provide some evidence against the possibility that it arises in synaptic depression at the geniculo-cortical synapse.

\section{Model of neural response and adaptation}

The model (Eqs. 1, 2; Fig. 1) stipulates three mechanisms that are desensitized by prolonged stimulation: an early stage mechanism that is untuned for orientation, a spatio-temporally tuned mechanism and a normalization stage that is untuned because it pools over a large number of tuned units, though it could also be supported by subcortical inputs. The early stage and the normalization pool are equally sensitive to all orientations and sensitive to a broad range of temporal frequencies. The temporal frequency sensitivity of the early stage and the normalization pool overlap, though are in general not the same. Both will be desensitized by low temporal frequency stimuli and high temporal frequency stimuli, but to different degrees. The tuned mechanism is desensitized only by stimuli of low temporal frequency at the preferred orientation.

Adaptation to any stimulus, when effective, almost always reduced contrast gain, reflected by a rightward horizontal shift of the contrast response function on logarithmic axes. Model simulations show that this occurs only if the early stage and the normalization pool are equally desensitized (Fig. 4). Unequal desensitization of these two mechanisms results in a combined change in contrast gain and response gain. It is important that an adapter can have different effects on the early stage and the nor- malization pool; otherwise our model could not account for response sensitization and response gain increases we see in the data after prolonged stimulus exposure. Our findings parallel those reported by Tailby et al. (2008) in the chromatic domain, suggesting that the adaptable mechanisms identified here are general features of cortical neurons. Because exposure to the low temporal frequency null adapters often resulted in increases in response gain and decreases in contrast gain, we infer that the normalization pool is more susceptible to desensitization than is the early stage. That the same cells might then exhibit reductions in response gain after exposure to low temporal frequency adapters at the preferred orientation we attribute to desensitization of the tuned mechanism sometimes being strong enough to countermand the increased response gain caused by desensitization of the normalization pool. When tuned adaptation is slight, cells can exhibit increased responses to high contrast stimuli following adaptation at the preferred orientation (Fig. 7; Sclar et al., 1989).

\section{Magnocellular contribution to cortical neural response}

Solomon et al. (2004) showed that subcortical neurons in the Mpathway were adapted by stimuli modulated at rates above the temporal passband of most cortical neurons. To the extent that this constitutes the adaptable early stage we can estimate the variation in the M-input among cells from the $A_{\mathrm{ES}}$ parameter of our model.

The high contrast sensitivity (low $\sigma$ ) of the cells with the largest presumed M-input implies that contrast sensitivity of cortical neurons depends on the relative strength of $\mathrm{M}$-influence, in agreement with Allison et al. (2000). Adaptation in the most sensitive neurons tends to increase response gain, suggesting that the neurons with the strongest $\mathrm{M}$-input also have the strongest normalization pools, and that the M-pathway contributes disproportionately to the normalization pool. Cells with the strongest inferred M-input also showed the greatest surround suppression. This is consistent with the strong normalization seen in retinal and geniculate M-pathway neurons (Solomon et al., 2006; Alitto and Usrey, 2008). The cells with presumed strong $\mathrm{M}$-input were not in other respects distinguished by their stimulus preferences. Sensitivity to high temporal frequencies is the other obvious characteristic one might expect of M-recipient V1 neurons. However, we explicitly excluded such neurons from our sample because we would have been unable to restrict adaptation to the untuned mechanisms if the cortical neurons under study responded to high temporal frequency adapters. The exclusion of these neurons likely explains why we did not find a significant correlation between presumed M-input and temporal sensitivity of the neurons we characterized.

\section{Relationship to psychophysics}

One of the advantages of this study was that we were able to maximally desensitize the neural mechanisms that contribute to a cortical neuron's response and study the neuron in a consistent adaptation state like that used in classical psychophysical studies. Adaptation to patterns modulated at high temporal frequencies elevates detection thresholds, regardless of pattern orientation (Kelly and Burbeck, 1987). This can be understood to reflect the desensitization of untuned mechanisms. Our finding that adaptation to these stimuli often increases response gain and sometimes increases the maximum response suggests (paradoxically) that contrast discrimination might be easier, and the apparent contrast of suprathreshold stimuli might be increased, following adaptation to high temporal frequency stimuli. We would also expect that prolonged exposure to low temporal frequency 
stimuli will lower contrast discrimination thresholds for highcontrast stimuli at the orthogonal orientation.

\section{References}

Albrecht DG, Hamilton DB (1982) Striate cortex of monkey and cat: contrast response function. J Neurophysiol 48:217-237.

Albrecht DG, Farrar SB, Hamilton DB (1984) Spatial contrast adaptation characteristics of neurones recorded in the cat's visual cortex. J Physiol 347:713-739.

Alitto HJ, Usrey WM (2008) Origin and dynamics of extraclassical suppression in the lateral geniculate nucleus of the macaque monkey. Neuron 57:135-146.

Allison JD, Melzer P, Ding Y, Bonds AB, Casagrande VA (2000) Differential contributions of magnocellular and parvocellular pathways to the contrast response of neurons in bush baby primary visual cortex (V1). Vis Neurosci 17:71-76.

Blakemore C, Campbell FW (1969) On the existence of neurones in the human visual system selectively sensitive to the orientation and size of retinal images. J Physiol 203:237-260.

Bonds AB (1989) Role of inhibition in the specification of orientationselectivity of cells in the cat striate cortex. Vis Neurosci 2:41-55.

Camp AJ, Tailby C, Solomon SG (2009) Adaptable mechanisms that regulate the contrast response of neurons in the primate lateral geniculate nucleus. J Neurosci 29:5009-5021.

Carandini M, Ferster D (1997) A tonic hyperpolarization underlying contrast adaptation in cat visual cortex. Science 276:949-952.

Carandini M, Heeger DJ, Movshon JA (1997) Linearity and normalization in simple cells of the macaque primary visual cortex. J Neurosci 17:8621-8644.

Carandini M, Movshon JA, Ferster D (1998) Pattern adaptation and crossorientation interactions in the primary visual cortex. Neuropharmacology 37:501-511.

Crowder NA, Price NS, Hietanen MA, Dreher B, Clifford CW, Ibbotson MR (2006) Relationship between contrast adaptation and orientation tuning in V1 and V2 of cat visual cortex. J Neurophysiol 95:271-283.

DeAngelis GC, Robson JG, Ohzawa I, Freeman RD (1992) Organization of suppression in receptive fields of neurons in cat visual cortex. J Neurophysiol 68:144-163.

Derrington AM, Lennie P (1984) Spatial and temporal contrast sensitivities of neurones in lateral geniculate nucleus of macaque. J Physiol 357:219-240.

Freeman TC, Durand S, Kiper DC, Carandini M (2002) Suppression without inhibition in visual cortex. Neuron 35:759-771.

Graham N (1989) Visual pattern analyzers. New York: Oxford UP.

Gur M, Kagan I, Snodderly DM (2005) Orientation and direction selectivity of neurons in V1 of alert monkeys: functional relationships and laminar distributions. Cereb Cortex 15:1207-1221.

Hammond P, Pomfrett CJ, Ahmed B (1989) Neural motion after-effects in the cat's striate cortex: orientation selectivity. Vision Res 29:1671-1683.

Hawken MJ, Parker AJ (1984) Contrast sensitivity and orientation selectivity in lamina IV of the striate cortex of old world monkeys. Exp Brain Res 54:367-372.

Hawken MJ, Shapley RM, Grosof DH (1996) Temporal-frequency selectivity in monkey visual cortex. Vis Neurosci 13:477-492.

Heeger DJ (1992) Normalization of cell responses in cat striate cortex. Vis Neurosci 9:181-197.
Hubel DH, Wiesel TN (1959) Receptive fields of single neurones in the cat's striate cortex. J Physiol 148:574-591.

Hubel DH, Wiesel TN (1962) Receptive fields, binocular interactions, and functional architecture in the cat's visual cortex. J Physiol 160:106-154.

Kelly DH, Burbeck CA (1987) Further evidence for a broadband, isotropic mechanism sensitive to high-velocity stimuli. Vision Res 27:1527-1537.

Kohn A (2007) Visual adaptation: physiology, mechanisms, and functional benefits. J Neurophysiol 97:3155-3164.

Maffei L, Fiorentini A, Bisti S (1973) Neural correlate of perceptual adaptation to gratings. Science 182:1036-1038.

Maffei L, Berardi N, Bisti S (1986) Interocular transfer of adaptation after effect in neurons of area 17 and 18 of split chiasm cats. J Neurophysiol 55:966-976.

Marlin SG, Hasan SJ, Cynader MS (1988) Direction-selective adaptation in simple and complex cells in cat striate cortex. J Neurophysiolgy 59:1314-1330.

Morrone MC, Burr DC, Maffei L (1982) Functional implications of crossorientation inhibition of cortical visual cells. I. Neurophysiological evidence. Proc R Soc Lond B 216:335-354.

Movshon JA, Blakemore C (1973) Orientation specificity and spatial selectivity in human vision. Perception 2:53-60.

Movshon JA, Lennie P (1979) Pattern-selective adaptation in visual cortical neurones. Nature 278:850-852.

Ohzawa I, Sclar G, Freeman RD (1985) Contrast gain control in the cat's visual system. J Neurophysiol 54:651-667.

Priebe NJ, Ferster D (2006) Mechanisms underlying cross-orientation suppression in cat visual cortex. Nat Neurosci 9:552-561.

Sanchez-Vives MV, Nowak LG, McCormick DA (2000a) Membrane mechanisms underlying contrast adaptation in cat area 17 in vivo. J Neurosci 20:4267-4285.

Sanchez-Vives MV, Nowak LG, McCormick DA (2000b) Cellular mechanisms of long-lasting adaptation in visual cortical neurons in vitro. J Neurosci 20:4286-4299.

Saul AB, Cynader MS (1989a) Adaptation in single units in visual cortex: the tuning of aftereffects in the spatial domain. Vis Neurosci 2:593-607.

Saul AB, Cynader MS (1989b) Adaptation in single units in visual cortex: the tuning of aftereffects in the temporal domain. Vis Neurosci 2:609-620.

Sclar G, Lennie P, DePriest DD (1989) Contrast adaptation in striate cortex of macaque. Vision Res 29:747-755.

Skottun BC, De Valois RL, Grosof DH, Movshon JA, Albrecht DG, Bonds AB (1991) Classifying simple and complex cells on the basis of response modulation. Vision Res 31:1079-1086.

Solomon SG, Peirce JW, Dhruv NT, Lennie P (2004) Profound contrast adaptation early in the visual pathway. Neuron 42:155-162.

Solomon SG, Lee BB, Sun H (2006) Suppressive surrounds and contrast gain in magnocellular-pathway retinal ganglion cells of macaque. J Neurosci 26:8715-8726.

Tailby C, Solomon SG, Dhruv NT, Lennie P (2008) Habituation reveals fundamental chromatic mechanisms in striate cortex of macaque. J Neurosci 28:1131-1139.

Vautin RG, Berkley MA (1977) Responses of single cells in cat visual cortex to prolonged stimulus movement: neural correlates of visual aftereffects. J Neurophysiol 40:1051-1065. 